\title{
Analytical techniques with a cyclotron on polymers
}

\author{
M P DE JONG*, M J A DE VOIGT, L J VAN IJZENDOORN and H H BRONGERSMA ${ }^{\dagger}$ \\ Cyclotron Laboratory and ${ }^{\dagger}$ Laboratory of Surfaces and Interfaces, Department of Applied Physics and Dutch \\ Polymer Institute, Eindhoven University of Technology, P.O. Box 513, 5600 MB Eindhoven, The Netherlands
}

\begin{abstract}
Nuclear analytical techniques (PIXE, RBS and ERDA) have been applied to investigate diffusion processes in polymer light-emitting diodes (PLEDs), employing a $30 \mathrm{MeV}$ AVF cyclotron. The techniques prove to be useful in identifying the origin of the diffusion process and the nature of molecules transported from the hole injecting ITO electrode into the polymer layer.
\end{abstract}

Keywords. Nuclear analytical techniques; cyclotron; polymers.

\section{Introduction}

Nuclear analytical methods appear to be useful for elemental analysis of polymers because of their quantitative character, generally high sensitivity and depth sensitivity. A disadvantage is the radiation damage induced by the primary beam. However, this damage can be either monitored or suppressed by sample cooling and both quantification and depth profiling are still possible without sputtering techniques.

Recent progress in polymer technology has led to the discovery of bright emission of light by PLEDs (Burroughes et al 1990). Also polymer based FETs and Schottky diodes have been developed (Brown et al 1997). The semiconducting properties and the long-term stability depend on many fundamental mechanisms related to the characteristics of mesoscopic structures and processes. If those mechanisms and structures can be identified and controlled, appreciable progress may be expected to realize large-scale production of polymer devices. Many applications would concern simple data storage, like chipcards, identification labels and very thin computer and television screens. The methods of fabrication are expected to be simple and cheap.

The electrical properties of the semiconducting polymer determine to a large extent the quantum efficiency and in this respect unintentional doping of the polymer should be minimized. In this paper diffusion processes of impurities into the polymer are discussed which influence the performance of PLEDs. We concentrate on diffusion of indium out of the indium tin oxide (ITO) contact into the polymer layer. The polymer layer consists of poly-( $p$-phenylene vinylene), or shortly PPV. Two different routes for preparing PPV layers exist. The most common route is to obtain PPV after thermal conversion of a chlorine-containing precursor. During the conversion from the precursor to $\mathrm{PPV}, \mathrm{HCl}$ is freed and evaporates

*Author for correspondence out of the layer. However, this $\mathrm{HCl}$ may also react with the ITO electrode and consequently $\mathrm{InCl}_{3}$ might appear as an impurity in the PPV. A second route to produce PPV layers is the application of soluble $\mathrm{OC}_{1} \mathrm{C}_{10}-\mathrm{PPV}$ that is spincoated onto a substrate; no further conversion is required and no $\mathrm{HCl}$ is involved.

Three nuclear analytical techniques suitable for the analysis of polymers will be discussed. Rutherford backscattering spectrometry (RBS) is applied to analyse heavy elements and elastic recoil detection analysis (ERDA) for light elements like hydrogen, carbon, oxygen and nitrogen. Both techniques provide depth sensitivity with resolutions of about $5 \mathrm{~nm}$ at the surface. For a lateral scan of the elemental distribution a $3 \mathrm{MeV}$ proton microprobe is employed with a resolution of about 3 micrometres for particle induced X-ray emission (PIXE); note that the latter technique is not depth sensitive. Alpha-particle beams are employed for RBS with energies up to $4 \mathrm{MeV}$ and for ERDA up to $16 \mathrm{MeV}$. A $30 \mathrm{MeV}$ light-ion AVF cyclotron produces the beams. Special beam optics are applied, like a dipersive magnetic system, to reduce the energy spread. High-energy light ions (protons and $\mathrm{He}^{++}$) have the advantage that their stopping powers in material are relatively small, and therefore they do not cause so much damage.

\section{Experimental}

\subsection{ERDA experiments}

The ERDA technique is well suited to investigate light elements, such as $\mathrm{H}, \mathrm{C}, \mathrm{N}, \mathrm{O}$, in a heavy matrix. With a light-particle beam $\left(\mathrm{He}^{++}\right)$discrimination between heavy recoils and elastically scattered alpha particles must be made. We developed a method using partially depleted $\mathrm{Si}$ detectors of the PIPS type (Canberra) to discriminate between scattered projectiles and recoils. The thickness of the depletion layer was tuned with the bias voltage 
such that the heavy recoils were stopped in the depletion layer and the alpha particles behind it. This gives rise to a fast timing signal in the former case and a slow one in the latter (Klein et al 1992).

This technique was applied to investigate the oxygen concentration in $\mathrm{OC}_{1} \mathrm{C}_{10}-\mathrm{PPV}$. The sample consisted of a PPV layer, $120 \mathrm{~nm}$ in thickness, deposited onto a silicon substrate and separated by a thin gold layer. A $13.4 \mathrm{MeV}$ alpha-particle beam hit the surface of the sample at a glancing angle. The recoils were detected in forward direction at an angle of $30^{\circ}$ with respect to the beam direction (see insert of figure 1). The data are collected in a scatter plot that represents the intensity vs energy of the particle and time of the timing signals (figure 1). The strong signal of scattered alpha particles leaves the weak signals of the heavy recoils completely free because of the slow timing of the alpha particles. A projection on the energy axis (figure 2 ) reveals the depth profiles of $\mathrm{C}$ and $\mathrm{O}$. The profiles are essentially background free. The oxygen signal stems from native covalently bound oxygen atoms in the PPV as well as from traces of oxygen originating from the ambient. This oxygen impurity should be minimized to prevent (photo) oxidation of the PPV, which degrades the conjugated backbone of the semiconducting PPV molecules. To discriminate between impurities from the ambient and the native oxygen, it is considered to perform both synthesis and operation under ${ }^{18} \mathrm{O}$ atmosphere in future. Quantification is then possible and can be carried out by using a calibration sample with known ${ }^{18} \mathrm{O}$ content.

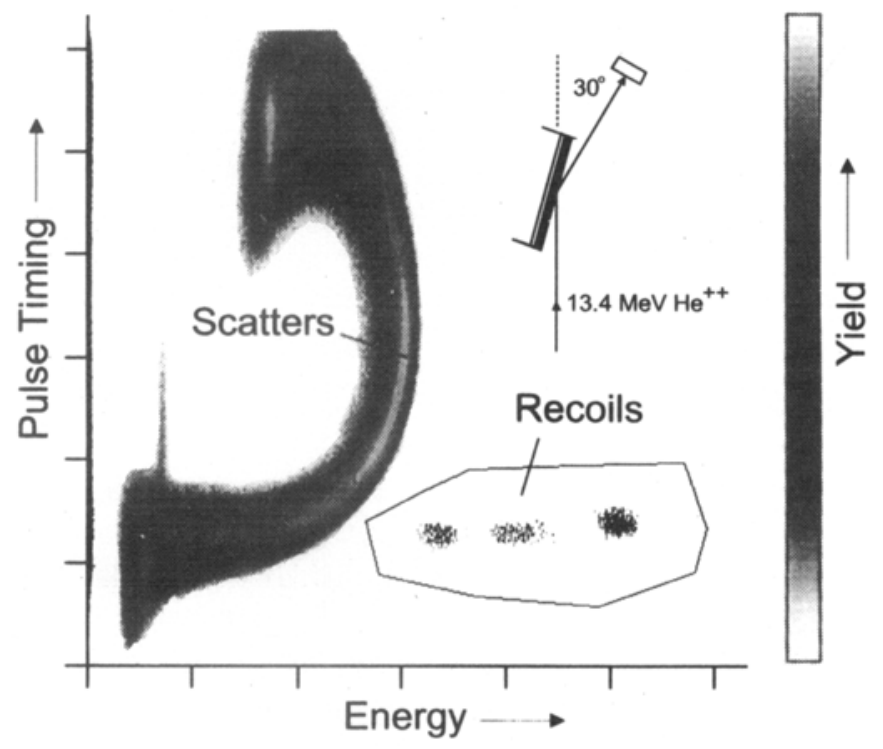

Figure 1. Scatter plot of the pulse-timing signal vs energy with intensity indicated by the grey scale on the right. The dark curve at the left is due to elastically scattered alpha particles and the heavy recoils are indicated by the contour.

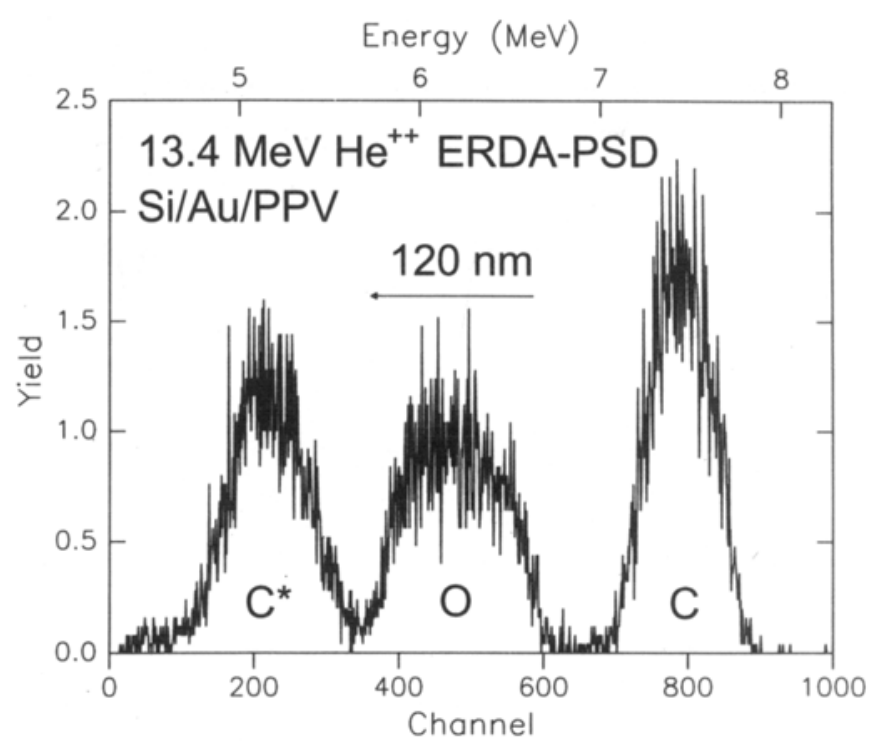

Figure 2. Projection of the signals of the heavy recoils (within the contour of figure 1 ) on the energy axis. Here $C^{*}$ indicates the peak due to carbon recoils that are excited to the first excited state. The oxygen profile corresponds to the $120 \mathrm{~nm}$ PPV layer.

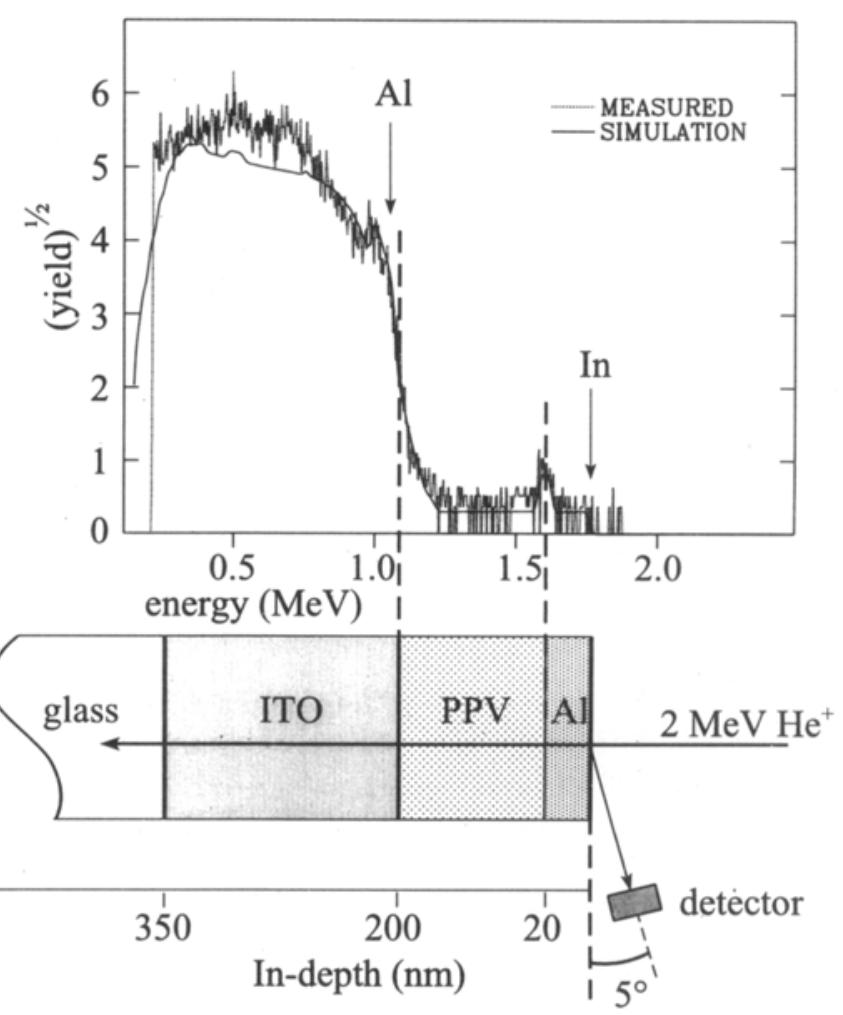

Figure 3. RBS spectrum of a PPV layer with ITO and Al contacts. The arrows indicate 'surface' energies of $\mathrm{Al}$ and In. The simulation has been carried out with the RUMP code. 


\subsection{RBS experiments}

The method of RBS is very well suited for depth profiling of heavy elements, e.g. the In and $\mathrm{Sn}$ of the ITO layer in a PPV film formed by the thermal conversion process. A sample has been prepared on a glass substrate with an ITO layer of $150 \mathrm{~nm}$. On top a PPV layer, $180 \mathrm{~nm}$ thick, was deposited covered with a $20 \mathrm{~nm}$ thick patterned $\mathrm{Al}$ layer. A $2 \mathrm{MeV} \mathrm{He}^{+}$beam did hit the sample perpendicular to the surface and the scattered particles were detected at a glancing angle of $5^{\circ}$ with the surface (see figure 3 ).

First, RBS spectra showed In diffusion in the uncovered PPV layer. A quantitative analysis was carried out using the RUMP simulation code (Doolittle 1986) which revealed an amount of 0.01 at \% of In in the PPV. After heating for $19 \mathrm{~h}$ up to $230^{\circ} \mathrm{C}$, the diffused amount of In increased to 0.1 at \%. The part of the PPV located under the $\mathrm{Al}$ layer showed accumulation of In at the PPV-Al interface. This is clearly seen in figure 3 where the maximum energy of backscattered particles from In atoms located in the ITO is $1.1 \mathrm{MeV}$ while the arrows indicate ions scattered from $\mathrm{Al}$ and In atoms located at the surface. The RUMP simulation showed that the detected ions with energy between 1.1 and $1.6 \mathrm{MeV}$ are due to In. It thus can be concluded that a significant amount of In out of the ITO contact layer diffused into the PPV, possibly causing deterioration of the LED device. To investigate whether In diffuses as single atom or as part of an $\mathrm{InCl}_{3}$ molecule possibly formed during the thermal conversion (Sauer et al 1995), PIXE analyses have been carried out employing a micro proton beam, as discussed below.

\subsection{Micro beam PIXE experiments}

The Eindhoven micro proton beam of $3 \mathrm{MeV}$ can be focussed with a Russian quadruplet magnet system. The obtained beam spot is approximately $3 \mu \mathrm{m}$ in the present PIXE analysis. For this analysis an $\mathrm{OC}_{1} \mathrm{C}_{10}-\mathrm{PPV}$ layer has been prepared that was treated in an $\operatorname{argon} / \mathrm{HCl}$ atmosphere to confirm the hypothesis that the ITO electrode erodes during exposure to $\mathrm{HCl}$ (Moratti 1998). The PIXE spectrum of the PPV film peeled of the substrate (figure 4) exhibits both $\mathrm{Cl}$ and In lines. A micro beam scan of an area of $360 \times 360 \mu \mathrm{m}$ reveals $\mathrm{Cl}$ and In distributions that are clearly correlated. A calibration run on an $\mathrm{InCl}_{3}$ sample produced a PIXE spectrum with $\mathrm{Cl}$ and In peaks having almost the same intensity ratio as for the PPV sample. From this we concluded that In diffuses as a $\mathrm{InCl}_{3}$ molecule through the PPV layer.
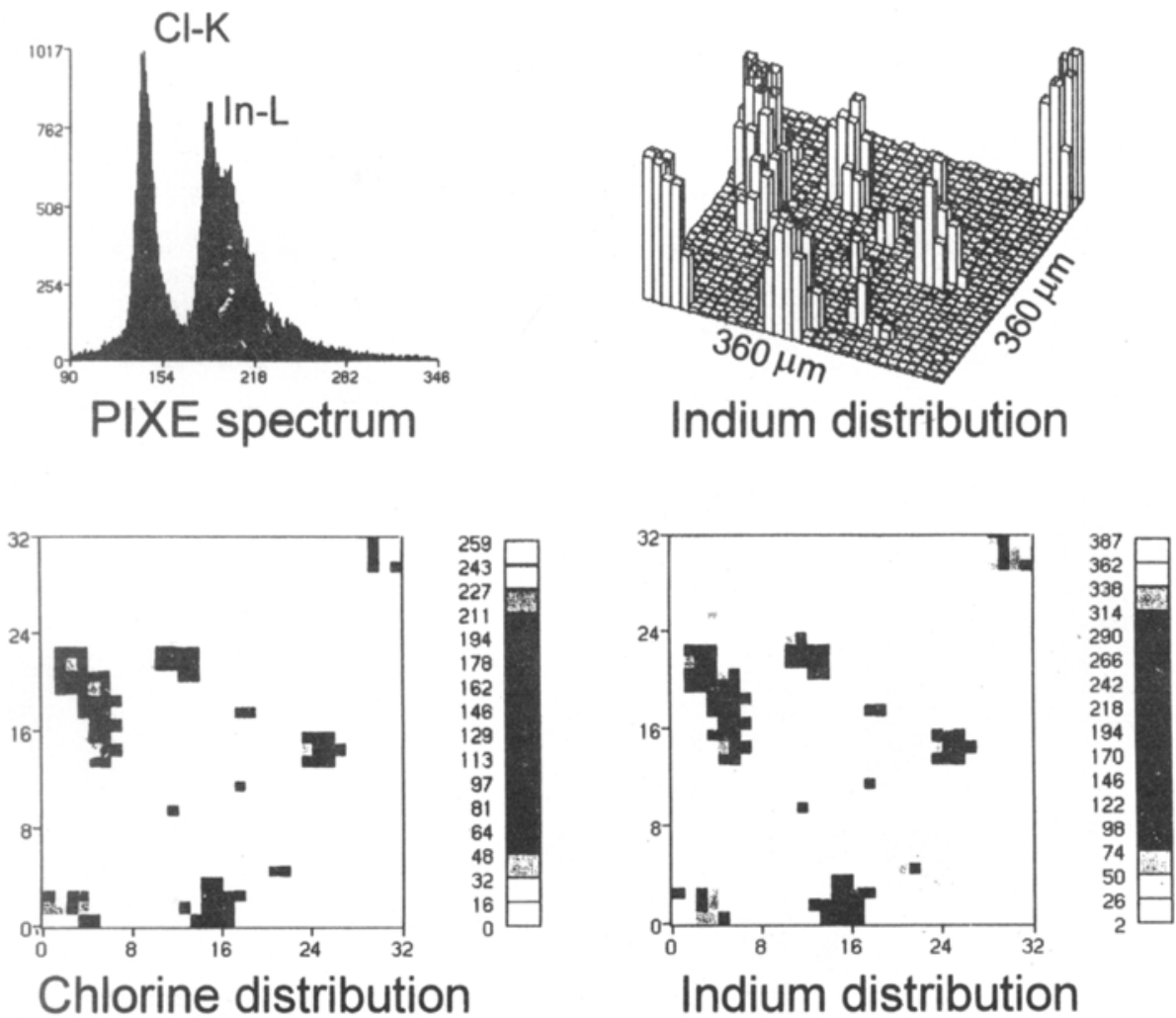

Figure 4. A PIXE spectrum (upper left) of a thin PPV layer and the corresponding In distribution of a lateral scan with the $3 \mu \mathrm{m}$ proton beam (upper right). The In and $\mathrm{Cl}$ distributions are compared at the bottom. 


\section{Discussion and conclusions}

The applied ion-beam analysis techniques appeared to cause some radiation damage to the polymer samples. Therefore the hydrogen yield was monitored during the experiments (de Jong et al 1997). Knowledge of the decrease with dose offered the possibility to correct for damage. We presently have installed a cooled-target facility that is able to freeze, with a temperature of $10 \mathrm{~K}$, the elements at its original position. In this way we expect to circumvent the inaccuracies introduced by radiation damage.

It is concluded that nuclear analytical methods provide sensitive and quantitative analyses with a depth resolution in the nm range of functional polymer layers. The ERDA technique is useful to analyse traces of light elements in a heavy matrix with depth resolutions down to a few nanometers at the surface. The RBS technique finds its strengths for heavy elements with about the same properties as ERD. The micro-beam PIXE technique provides lateral resolution in the $\mu \mathrm{m}$ range. The combination of techniques applied to PLED analyses, leads to detailed insight in diffusion processes revealing the sensitivity of the PLED for the preparation techniques. A cyclotron can be used fruitfully if the beam quality is improved. This concerns improvement of the beam spread, and thus of beam-energy resolution, by a factor of three to about $10^{-3}$. It then offers the unique combination of high resolution and high beam energy, needed for the light-ion ERDA technique.

\section{References}

Brown A R, Jarrett C P, de Leeuw D M and Matters M 1997 Synth. Metals 8837

Burroughes J H, Bradley D D, Brown A R, Marks R N, Mackey K, Friend R H, Burn P L and Holmes A B 1990 Nature 347539

De Jong M P, Maas A J H, van Ijzendoorn L J, Klein S S and de Voigt M J A 1997 J. Appl. Phys. 821058

Doolittle L R 1986 Nucl. Instr. \& Meth. B15 227

Klein S S and Rijken H A 1992 Nucl. Instr. \& Meth. B66 393

Moratti S C 1998 in Handbook of conducting polymers (eds) T A Skotheim, R L Elsenbaumer and J R Reynolds (New York: Marcell Dekker Inc.) pp 343-361

Sauer G et al 1995 J. Anal. Chem. 353642 